\title{
ELASTIC STABILITY OF 3D IDEAL TRUSSES
}

\author{
J.M. ROESSET \\ Texas A\&M University, Austin, USA.
}

\begin{abstract}
The contents of this paper reflect work that was conducted in the early 1960s in preparation of class notes for a graduate course on Elastic Stability taught in the Civil Engineering Department of MIT. This material is now well known and the presentation is not submitted as a new or original contribution but rather as a reflection of some work done that is related to the teachings of Professor Jerry Connor when he started at MIT. It is intended as an example of Jerry's impact since his early years on generations of students and young colleagues and an acknowledgement of our debt to him.
\end{abstract}

\section{INTRODUCTION}

When Jerry Connor started teaching at MIT, I had already completed all my course work and I was working on my doctoral thesis. I did not therefore take any courses from him. I benefited greatly, however, from his advice and support both as a graduate student and as an assistant professor, my conversations with him and my contacts with the graduate students he was supervising.

Until then we had learned matrix Structural Analysis through a rather complicated electrical analogy that did not make much physical sense to a structural engineer. One had to form an incidence matrix $\mathrm{A}$, a matrix $\mathrm{K}$ and multiply the transpose of $\mathrm{A}$ by $\mathrm{K}$ and $\mathrm{A}$ in order to form the total stiffness matrix of the structure. Jerry started teaching it using the direct stiffness method, much simpler and much more logical, forming the stiffness matrix of each member and then assembling them. While everything presented in this paper is well known now and there is no claim of any original material, in the early 1960s a distinction was made between eigenvalue, or bifurcation buckling, and nonlinear or limit point instability. It was postulated that these two different phenomena required entirely different formulations. Jerry showed me that both of them could be derived from a unique general formulation although one may later make different assumptions and simplifications to determine the buckling load. I used this in writing my class notes for the graduate course on Structural Stability that I taught at MIT. The simplest case, where one can actually obtain explicit closed form expressions for the secant and tangent stiffness matrices of each member and thus the complete structure is that of an ideal truss. This paper reproduces the general formulation of the equilibrium and stability problems for a three dimensional ideal truss, something I had not seen published anywhere at that time, with application to the well-known and very simple case of a two bar truss., showing that both types of instability can be obtained from the same formulation. The objective is to study the overall stability of ideal space trusses such as lattice towers or truss domes, without consideration of the local stability of the individual members and with all the classical simplifying assumptions of ideal trusses.

\section{GENERAL CONSIDERATIONS}

The equilibrium equations for a truss structure can be derived directly enforcing at each joint the equilibrium of internal bar forces and the applied loads in the deformed position of the structure or making stationary the first variation of the total potential energy. Whether the 
equilibrium is stable, unstable or indifferent will depend on the sign of the second variation. Buckling is normally defined as the case when this second variation is null. Whether the equilibrium is stable or unstable will depend then on the value of the third variation along the direction of the buckling shape [1]. This may require in some cases looking also at the fourth variation.

Calling D the vector of bar elongations for an ideal truss, $U$ the vector of joint displacements, $\mathrm{P}$ the vector of applied joint loads and $\mathrm{K}$ a diagonal matrix with the stiffness (EA/L) of each bar as the diagonal elements, the first variation of the total potential energy can be written as

$$
\delta \Pi=\delta D^{T} K D-\delta U^{T} P
$$

and thus the equilibrium equations will result from

$$
\delta D^{T} K D=\delta U^{T} P
$$

Expressing the elongations in terms of the displacements, $U$ the equations can be rewritten as

$$
\delta \mathrm{U}^{\mathrm{T}} \mathrm{S}_{\mathrm{sec}}(\mathrm{U}) \mathrm{U}=\delta \mathrm{U}^{\mathrm{T}} \mathrm{P}
$$

or

$$
\mathrm{S}_{\mathrm{sec}}(\mathrm{U}) \mathrm{U}=\mathrm{P}
$$

where $S_{\text {sec }}$ would be the secant stiffness matrix of the structure whose terms are functions of the unknown displacements U.

Since these are nonlinear equations, there is an infinite number of ways in which terms can be reordered to form equivalent secant stiffness matrices. Since it is not unique, the secant stiffness matrix will not be symmetric in general but one can always find an equivalent form that is symmetric.

The second variation of the total potential energy is given by

$$
\delta^{2} \Pi=\delta^{2} \mathrm{U}^{\mathrm{T}} \mathrm{S}_{\mathrm{sec}}(\mathrm{U}) \mathrm{U}+\delta \mathrm{U}^{\mathrm{T}}\left(\delta \mathrm{S}_{\mathrm{sec}}(\mathrm{U}) \mathrm{U}+\mathrm{S}_{\mathrm{sec}}(\mathrm{U}) \delta \mathrm{U}\right)-\delta^{2} \mathrm{U}^{\mathrm{T}} \mathrm{P}-\delta \mathrm{U}^{\mathrm{T}} \delta \mathrm{P}
$$

At an equilibrium position, $\mathrm{S}_{\text {ecc }}(\mathrm{U}) \mathrm{U}=\mathrm{P}$ and therefore the two terms with the second variation of the displacements cancel each other. As a result, the incremental (tangent) equilibrium equations become

$$
\delta \mathrm{S}_{\mathrm{sec}}(\mathrm{U}) \mathrm{U}+\mathrm{S}_{\mathrm{sec}}(\mathrm{u}) \delta \mathrm{U}=\delta \mathrm{P}
$$

Or rearranging the terms involving the first variation of the displacements

$$
\mathrm{S}_{\tan }(\mathrm{U}) \delta \mathrm{U}=\delta \mathrm{P}
$$

where $S_{\tan }(U)$ is the tangent stiffness matrix at an equilibrium position. The terms of this matrix are functions of the displacements in the deformed equilibrium position but the unknowns are the incremental or differential displacements from that position. The stability condition is then for the tangent stiffness matrix to be positive definite (all eigenvalues positive). Indifferent equilibrium or buckling is reached when the tangent stiffness matrix becomes singular, or what is the same when it is positive semi definite with a zero eigenvalue. The eigenvector corresponding to that null eigenvalue would then be the buckling shape.

It has been said that the tangent stiffness matrix will not be symmetric in general. This would be so if it were evaluated at a position that does not correspond exactly to an equilibrium 
condition between the internal forces and the applied loads. This would happen if one were to perform an incremental analysis without iterating at each step to reach exactly a position of equilibrium. On the other hand if the tangent stiffness matrix is defined at an exact equilibrium position, it will be symmetric.

The secant and tangent stiffness matrices of a truss member can be written in the form

$$
\left[\begin{array}{cc}
S & -S \\
-S & S
\end{array}\right]
$$

where $S$ would be a 3 by 3 matrix for a space truss and a 2 by 2 matrix for a plane truss. In the following, the derivation of the secant and tangent stiffness matrices will be concerned only with the matrix $\mathrm{S}$. The total stiffness matrices of each member will result from the above expression and those of the complete structure would be obtained assembling the member matrices with the direct stiffness method.

\section{3D IDEAL TRUSSES}

Consider a bar of an ideal space truss in the original and in the deformed position. Calling xi ( $i=1-3)$ the coordinates of a point (joint) in a system of 3 orthogonal coordinate axes, and ui $(i=1-3)$, the displacements of a joint along the same direction if the member connects joints $m$ and $n$ of the truss defining

$$
\Delta \mathrm{x}_{\mathrm{i}}=\mathrm{x}_{\mathrm{i}, \mathrm{n}}-\mathrm{x}_{\mathrm{i}, \mathrm{m}} \quad \Delta_{\mathrm{ui}}=\mathrm{u}_{\mathrm{i}, \mathrm{n}}-\mathrm{u}_{\mathrm{i}, \mathrm{m}}
$$

The director cosines of the bar in the original position are

$$
\cos \alpha_{i}=\Delta x_{i} / L
$$

where $\mathrm{L}$ is the original length of the bar, and in the deformed position

$$
\cos \beta_{\mathrm{i}}=\left(\Delta \mathrm{x}_{\mathrm{i}}+\Delta \mathrm{u}_{\mathrm{i}}\right) /(\mathrm{L}+\mathrm{e})
$$

where e is now the bar elongation.

$$
\mathrm{e}=\Sigma \Delta \mathrm{u}_{\mathrm{i}}\left(\cos \alpha_{\mathrm{i}}+\cos \beta_{\mathrm{i}}\right) /(1+\cos \theta)
$$

with

$$
\cos \theta=\Sigma \cos \alpha_{i} \cdot \cos \beta_{i}
$$

Then

$$
\mathrm{de}=\Sigma \cos \beta_{\mathrm{i}} \cdot \mathrm{d}\left(\Delta \mathrm{u}_{\mathrm{i}}\right)
$$

and

$$
\mathrm{d}\left(\cos \beta_{\mathrm{i}}\right)=\left(\mathrm{d}\left(\Delta \mathrm{u}_{\mathrm{i}}\right)-\Sigma \cos \beta_{\mathrm{i}} \cdot \cos \beta_{\mathrm{j}} \cdot \mathrm{d}\left(\Delta \mathrm{u}_{\mathrm{j}}\right)\right) /(\mathrm{L}+\mathrm{e})
$$

The forces at the end of a member are $\mathrm{X}_{\mathrm{i}}=\mathrm{F}, \cos \beta_{\mathrm{i}}=\mathrm{EA}, \cos \beta_{\mathrm{i}} / \mathrm{L}$

The terms of the secant stiffness matrix are then given by

$$
\mathrm{S}_{\mathrm{sec} i, \mathrm{j}}=\mathrm{EA} \cos \beta_{\mathrm{i}}\left(\cos \alpha_{\mathrm{i}}+\cos \beta_{\mathrm{j}}\right) /(\mathrm{L}(1+\cos \theta))
$$

Clearly this matrix is not symmetric because of the terms $\cos \beta_{i} \cdot \cos \alpha_{j}$. Noticing however that

$$
\cos \beta_{\mathrm{i}} \cdot \cos \alpha_{\mathrm{j}} \cdot \Delta \mathrm{u}_{\mathrm{j}}=\left(\mathrm{L} \cos \alpha_{\mathrm{i}}+\Delta \mathrm{u}_{\mathrm{i}}\right) \cos \alpha_{\mathrm{j}} \cdot \Delta \mathrm{u}_{\mathrm{j}} /(\mathrm{L}+\mathrm{e})
$$


The terms can be rearranged so that

$$
\begin{aligned}
& S_{\text {sec } i, i}=\frac{E A}{L(1+\cos \theta)}\left(\cos \beta_{i}\left(\cos \alpha_{i} \cdot \cos \beta_{i}\right)+\sum_{j \neq i} \frac{\cos \alpha_{j} \cdot \Delta u_{j}}{L+e}\right) \\
& S_{\sec i, j}=\frac{E A}{L(1+\cos \theta)}\left(\frac{L}{L+e} \cos \alpha_{i} \cdot \cos \alpha_{j}+\cos \beta_{i} \cdot \cos \beta_{j}\right)
\end{aligned}
$$

and in this equivalent form, the secant matrix is symmetric. The summation in the expression for the diagonal terms extends over values of the subscript $j$ different from $i$.

The incremental, differential, forces at the ends of the member are

$$
\mathrm{dX}_{\mathrm{i}}=\mathrm{dF} \cos \beta_{\mathrm{i}}+\mathrm{Fd}\left(\cos \beta_{\mathrm{i}}\right)=\mathrm{EA}\left(\mathrm{de} \cos \beta_{\mathrm{i}}+\mathrm{ed}\left(\cos \beta_{\mathrm{i}}\right)\right) / \mathrm{L}
$$

and substituting the expressions for de and $\mathrm{d}\left(\cos \beta_{\mathrm{i}}\right)$, the terms of the tangent stiffness matrix for the member become

$$
\begin{aligned}
S_{t_{i, i}} & =\frac{E A}{L}\left(\cos ^{2} \beta_{i}+\frac{e}{L+e} \sin ^{2} \beta_{i}\right) \\
S_{t_{i, \mathrm{j}}} & =\frac{E A}{L+e} \cos \beta_{i} \cdot \cos \beta_{j}
\end{aligned}
$$

These expressions can be written alternatively as

$$
\begin{aligned}
S_{t_{i, i}} & =\frac{E A}{L} \cos ^{2} \beta_{i}+\frac{F}{L+e} \sin ^{2} \beta_{i} \\
S_{t_{i, j}} & =\frac{E A}{L} \cos \beta_{i} \cos \beta_{j}-\frac{F}{L+e} \cos \beta_{i} \cos \beta_{j}
\end{aligned}
$$

In this form the tangent stiffness matrix can be considered as the sum of 2 matrices: one corresponding to the normal stiffness matrix of a truss member but using the rotation terms (director cosines) in the deformed position, the second proportional to the force in the bar. It should be noticed that for elastic buckling of structures made of usual structural materials, the value of the elongation e should always be very small compared to the length of the bar and therefore the original length $\mathrm{L}$ can be used in these final expressions instead of the length in the deformed position $\mathrm{L}+\mathrm{e}$.

The rigorous determination of the buckling load requires then the solution of the nonlinear equilibrium equations to define the deformed geometry and the forces in the bar i that configuration, then the verification of whether the tangent stiffness matrix is positive definite in order to find the condition under which it becomes singular. This formulation will yield both the eigenvalue or bifurcation buckling and the limit point buckling. In each case however the simplifying approximations that can be used for the solution will be different. In a linearized stability analysis, the initial geometry or the deformed geometry obtained from a linear analysis will be used and the forces in the bars will be those resulting from the linear equilibrium solution. They will then be proportional to the applied loads. If one assumes that the loads are increased proportionally, the buckling load can be considered to be some initial value of the loads multiplied by a factor. The second part of the tangent stiffness matrix would be then proportional to this factor and the determination of the buckling load would involve the solution of a linear eigenvalue problem. The smallest eigenvalue would be the value of the factor corresponding to the buckling load. This approximate formulation will produce reasonable 
when the change in geometry due to the deformations under the buckling load is very small. It will produce erroneous results when the changes are relatively large. This tends to be the case for limit point, or snap through cases.

\section{APPLICATION TO A 2 BAR PLANE TRUSS}

Consider a symmetric 2 bar truss subjected to only a vertical load at the top. In this case

$\begin{array}{llll}\text { For bar } 1 \cos \alpha_{1}=\cos \alpha & \cos \alpha_{2}=\sin \alpha & \cos \beta_{1}=\cos \beta & \cos \beta_{2}=\sin \beta \\ \text { For bar } 2 \cos \alpha_{1}=\cos \alpha & \cos \alpha_{2}=\sin \alpha & \cos \beta_{1}=-\cos \beta & \cos \beta_{2}=\sin \beta\end{array}$

The total secant and tangent stiffness matrices of this case would then be 2 by 2 diagonal matrices because the off diagonal terms that correspond to the 2 bars cancel each other. Under a purely vertical load there would be only a vertical displacement $\mathrm{v}$ and the horizontal displacement $u$ would be null. The vertical nonlinear secant equilibrium equation becomes

$$
\frac{2 E A}{L(1+\cos \theta)} \sin \beta(\sin \alpha+\sin \beta) \mathrm{u}_{2}=X_{2}
$$

The tangent stiffness matrix of the complete truss is in this case a diagonal matrix because the contributions of the 2 bars to the off diagonal terms cancel each other while there are only vertical displacements. The 2 tangent equilibrium equations in the deformed position are

$$
\begin{aligned}
& \left(\frac{2 E A}{L} \cos ^{2} \beta+\frac{2 F}{L+e} \sin ^{2} \beta\right) d u_{1}=d X_{1} \\
& \left(\frac{2 E A}{L} \sin ^{2} \beta+\frac{2 F}{L+e} \cos ^{2} \beta\right) d u_{2}=d X_{2}
\end{aligned}
$$

The first equation represents the incremental (differential) horizontal equilibrium. If the coefficient of the $d u_{1}$ term vanishes, there would be a horizontal displacement without any horizontal load representing a bifurcation type buckling. This case could control if the angle $\alpha$ were close to 90 degrees. Assuming then that $\beta=\alpha$ (writing the equation in the undeformed original position, implying a negligible change in geometry prior to bucking, one would obtain the linearized solution

$$
F_{c r i}=-\frac{E A \cos ^{2} a}{\sin ^{2} \alpha}
$$

and

$$
P_{c r i}=2 F_{c r i} \sin \alpha=-\frac{2 E A \cos ^{2} \alpha}{\sin \alpha}
$$

If the coefficient of the second equation vanishes, the instability would occur in the vertical direction. This would occur for very small values of $\alpha$. In this case, the change in geometry prior to buckling would be relatively important and the linearized solution would not be valid.

$$
F_{c r i}=-E A \frac{L+e}{L} \frac{\sin ^{2} \beta}{\cos ^{2} \beta}
$$

and

$$
P_{c r i}=-2 E A \frac{L+e}{L} \frac{\sin ^{3} \beta}{\cos ^{2} \beta}
$$

In the elastic range for most materials $L+e$ can be considered equal to $L$. 


\section{CONCLUSIONS}

The objective of this paper was to present a set of derivations developed as class notes in the early 1960s when the author was a starting assistant professor at MIT and a colleague of Jerry Connor. With no attempt to present them as new or original material, the paper is intended as recognition of the influence of Jerry on his students and young colleagues. Jerry is a man of great vision who has cared only about his teaching, his research, and doing the best job at them, rather than his recognition, his number of publications, or the many other ways of counting beans devised by bureaucrats. The country and the world would greatly benefit from more Jerry Connors.

\section{REFERENCE}

[1] Connor, J.J., Analysis of Structural Member System, Ronald Press Company: New York, 1976. 